\title{
Research on Extension Evaluation of Rural Road Maintenance Quality Based on Game Theory
}

\author{
Gongliang Zhang ${ }^{1}$, Shou Chen ${ }^{*}$, Ping Xiao \\ ${ }^{1}$ School of Management, Zhongkai University of Agriculture and Engineering, Guangzhou, China \\ ${ }^{2}$ Faculty of Civil and Transportation Engineering, Guangdong University of Technology, Guangzhou, China \\ Email:*cs_gdut@163.com
}

How to cite this paper: Zhang, G.L., Chen, S. and Xiao, P. (2020) Research on Extension Evaluation of Rural Road Maintenance Quality Based on Game Theory. Applied Mathematics, 11, 670-677.

https://doi.org/10.4236/am.2020.117045

Received: July 3, 2020

Accepted: July 20, 2020

Published: July 23, 2020

Copyright () 2020 by author(s) and Scientific Research Publishing Inc. This work is licensed under the Creative Commons Attribution International License (CC BY 4.0).

http://creativecommons.org/licenses/by/4.0/ (c) (i) Open Access

\begin{abstract}
The importance of rural highway maintenance is self-evident, and it is also a basic work of the highway operation department. Selecting an appropriate evaluation method can timely and accurately find the pavement diseases, and improve the timeliness of pavement repair. To improve the accuracy of pavement quality comprehensive evaluation, based on the game theory, the improved extension evaluation method was combined with subjective and objective weight determination methods in this paper, to propose a comprehensive weight determination method with a good operability. The results show that the improved extension evaluation method provides a feasible scheme for rural highway maintenance quality evaluation.
\end{abstract}

\section{Keywords}

Rural Highway, Extension Evaluation, Comprehensive Weighting Method, Game Theory

\section{Introduction}

The development of rural highways has a positive practical significance, because it can provide key support for the smooth promotion of rural revitalization strategy. The maintenance of rural highways is the top priority, which will greatly influence the normal operation and sustainable development of the local social economy. Meanwhile, a scientific and reasonable evaluation method can be adopted to timely find the highway diseases and damages [1], so that effective measures can be taken to repair the pavement diseases and the operation ability and service status of rural highways can be guaranteed, thus providing a safe, 
fast and comfortable driving environment for travellers [2]. However, the evaluation coefficients in the traditional specifications [3] [4] don't have dynamic changes, so that the final evaluation results cannot objectively and accurately reflect the current situation of rural highway maintenance.

In the use of rural road maintenance quality evaluation methods, Yingchang Duan et al. [5] established a high-grade road maintenance quality assessment index system, using fuzzy multi-level evaluation algorithm to establish a comprehensive evaluation model, and verified the AHP-FUZZY algorithm in road maintenance evaluation through examples. The index has certain applicability; using extension theory, based on the supplement of related evaluation indexes, the matter element model of highway maintenance quality evaluation is established, which verifies the scientificalness and effectiveness of the method (Gongliang Zhang et al. [6]); Feizhen Huang [7] constructed a fuzzy extension evaluation model based on analytic hierarchy process and group integration empowerment. Combined with examples, it comprehensively evaluated the maintenance quality of rural road bridges, and provided a new method for comprehensive evaluation of rural highway bridge maintenance quality. As a comprehensive evaluation method [8] [9] [10], extension theory has been applied in many fields. However, the extension theory has few relevant applications in the evaluation of rural road maintenance quality. In addition, the extension method has some shortcomings in terms of integrity. Especially in the evaluation process, the weight determination has no fixed calculation model, which leads to limitations in the evaluation process.

In view of this, this paper uses the improved extension evaluation method to comprehensively evaluate the quality of rural road maintenance, and the game theory is used to combine the subjective weighting method (improved analytic hierarchy process) and the objective weighting method (simple correlation function method) to solve the problem of simple correlation function method which relies entirely on sample data to determine the index weight. The improved extension evaluation method has great advantages.

\section{Method for Determining Extension Evaluation Weight Coefficient Based on Game Theory}

The determination of the weight coefficient in the extension evaluation theory will greatly affect the results of comprehensive evaluation of multi-index road maintenance quality. The same index and data using the same calculation method to determine the weight will give different accuracy evaluation results. Choosing a scientific and reasonable method to determine the weight is the primary condition for improving the accuracy of the comprehensive evaluation results of the entire road maintenance quality. However, the existing extension evaluation theory does not have a fixed solution method for determining the index weight coefficient, generally, just the simple correlation function method is used to objectively weight each evaluation index, or only artificially subjectively 
determine the weights of each evaluation index, these two methods of determining weights have their advantages and disadvantages. Based on this, on the basis of objective weighting method and subjective weighting method, this paper adopts a comprehensive weighting method based on game theory to determine the weight coefficient. This method combines the subjective and objective weighting methods to obtain a balanced comprehensive weighting coefficient that can improve the accuracy of the comprehensive evaluation results of highway maintenance quality.

\subsection{Method for Determining Objective Weightings}

The objective weighting method is a method of determining the weight coefficient by using the objective information reflected by each evaluation index value from the actual data, and the method does not depend on the subjective judgment of the individual. In the traditional extension evaluation, the method of simple correlation function [11] is generally used to determine the objective weighting. This paper uses this method in determining the objective weighting coefficient. Assume:

$$
r_{i j}\left(v_{i}, V_{i j}\right)= \begin{cases}\frac{2\left(v_{i}-a_{j i}\right)}{b_{j i}-a_{j i}} & \left(v_{i} \leq \frac{b_{j i}+a_{j i}}{2}\right) \\ \frac{2\left(b_{j i}-v_{i}\right)}{b_{j i}-a_{j i}} & \left(v_{i} \geq \frac{b_{j i}+a_{j i}}{2}\right)\end{cases}
$$

$v_{i} \in V_{i p} \quad$ (Byte domain), that is

$$
r_{i j_{\max }}\left(v_{i}, V_{i j}\right)=\max _{j}\left\{r_{i j}\left(v_{i}, V_{i j}\right)\right\}
$$

If the category corresponding to the data of index $i$ is larger, the index will be given a larger weight value, then take:

$$
r_{i}= \begin{cases}j_{\max }\left[1+r_{i j_{\max }}\left(v_{i}, V_{i j}\right)\right] & \left(r_{i j_{\max }}\left(v_{i}, V_{i j}\right) \geq-0.5\right) \\ 0.5 j_{\max } & \left(r_{i j_{\max }}\left(v_{i}, V_{i j}\right)<-0.5\right)\end{cases}
$$

If the category corresponding to the data of index $i$ is larger, the index will be given a smaller weight value, then take:

$$
r_{i}= \begin{cases}\left(m-j_{\max }+1\right)\left[1+r_{i j_{\max }}\left(v_{i}, V_{i j}\right)\right] & \left(r_{i j_{\max }}\left(v_{i}, V_{i j}\right) \geq-0.5\right) \\ 0.5\left(m-j_{\max }+1\right) & \left(r_{i j_{\max }}\left(v_{i}, V_{i j}\right)<-0.5\right)\end{cases}
$$

Then the weight of index $i$ is:

$$
W_{i}=r_{i} / \sum_{i=1}^{n} r_{i}
$$

\subsection{Method for Determining Subjective Weightings}

The subjective weighting method, also known as the empirical weighting meth- 
od, is a method of relative weight based on the experience or preference of the decision makers and comparing the importance of each index. It has nothing to do with the distribution of attribute index. Common methods include the Delphi method, the Analytic Hierarchy Process (AHP), and the superiority chart method [11] [12] [13]. Among them, the AHP method is more commonly used, which constructs the judgment matrix by establishing the hierarchical relationship of each index, and then determines the weight of each index. In this paper, the improved analytic hierarchy process is used to determine the subjective weight coefficient. This method obtains the factor weights by self-correcting and self-coordinating the judgment matrix, then obtain the eigenvector of the consistent matrix and obtain the factor weights, which reduced the eigenvalues of the matrix and its consistency test process, simplified the analytic hierarchy process.

1) Sort the importance of evaluation index

By calculating the simple correlation function in Equation (1), the maximum simple correlation function value of each evaluation index and its associated quality level can be found separately. If the quality level is larger (the worse), it means that the evaluation index is more unfavorable to the final quality level, that is, more important than other indexes; if the maximum simple correlation function value of several indexes is associated with the same quality level, the more important the evaluation index of the simple correlation function is, the more important it is. According to this method, the importance of the quality assessment indexes for conservation is sorted.

2) Determine the judgment matrix

The judgment matrix is determined according to the order of importance of each maintenance quality evaluation index determined by the above method and the 1 - 9 scale system $A=\left(a_{i j}\right)_{n \times n}$.

3) Consistency test of judgment matrix

Consistency test is performed on the judgment matrix by using the deviation consistency index $\mathrm{CI}$, the random consistency index RI and the consistency ratio CR. Deviation from the consistency test index CI:

$$
\mathrm{CI}=\frac{\lambda_{\max }-n}{n-1}
$$

To determine the largest eigenvalue of the matrix, $\lambda_{\max }$ is the dimension of the matrix.

The value of the random consistency index RI varies with the dimension $n$ of the matrix. The specific changes are shown in Table 1.

Table 1. Random consistency index RI.

\begin{tabular}{cccccccccc}
\hline $\begin{array}{c}\text { Matrix } \\
\text { dimension n }\end{array}$ & 1 & 2 & 3 & 4 & 5 & 6 & 7 & 8 & 9 \\
\hline RI & 0 & 0 & 0.52 & 0.89 & 1.12 & 1.24 & 1.36 & 1.41 & 1.46 \\
\hline
\end{tabular}


Consistency ratio CR:

$$
\mathrm{CR}=\frac{\mathrm{CI}}{\mathrm{RI}}
$$

In general, the smaller the $\mathrm{CR}$, the better the consistency of the judgment matrix. When $\mathrm{CR}<0.1$, the consistency of the judgment matrix is within an acceptable range.

4) Correction of judgment matrix

Through the method in the literature (8), the self-coordination and self-correction of the judgment matrix were carried out to obtain a completely consistent matrix:

$$
A=b_{i j}=\frac{\sqrt[n]{\prod_{i=1}^{n} a_{i l}}}{\sqrt[n]{\prod_{i=1}^{n} a_{j l}}}
$$

5) Determination of weight coefficient

Use the geometric mean method (square root method). First calculate the geometric mean of the multiplication of the elements of each row of the judgment matrix, and then normalize the weight vector:

$$
\begin{gathered}
W=\left(W_{1}, W_{2}, \cdots, W_{i}\right) \\
W_{i}=\frac{\left(\prod_{j=1}^{n} b_{i j}\right)^{\frac{1}{n}}}{\sum_{i=1}^{n}\left(\prod_{j=1}^{n} b_{i j}\right)^{\frac{1}{n}}}, i=1,2, \cdots, n
\end{gathered}
$$

\subsection{Method for Determining Comprehensive Weight Based on Game Theory}

The method of determining the comprehensive weight based on game theory, first, we should briefly introduce the basic concepts of game theory, including participants, actions, information, strategy, payment, results and equilibrium. Among them, participants, strategy and payment are least needed to describe a game, so mainly introduce the rest several game elements, and the action and information are their "building blocks". Participants, actions, and results are collectively referred to as "rules of the game".

Participant: refers to a decision-making main body in a game whose purpose is to maximize the level of payment (utility) by selecting actions (or strategies). There are $L$ ways to empower index, and we see them as $L$ participants. Action: refers to the decision variable of the participant at a certain point in the game. In general, we use ai to indicate a specific action for the participant $i$, and $A_{i}=\left\{a_{i}\right\}$ denotes a set of all actions available for $i$ to choose. The action of the participants in this paper is to choose the weight $u_{k m}$.

Strategy: refers to the rules of action of a participant in the context of a given set of information, which stipulates when the participant chooses what action to 
take. The information set contains a participant's knowledge about the previous actions of other participants, and the strategy tells the participant how to respond to the actions of other participants. The weight vector of formula (11) in this paper represents the strategic combination of participants. Payment: refers to the level of defined utility that a participant receives under a particular strategy combination, or the level of expected utility that a participant receives. Payment is what the game participants really care about. The objective function of formula (12) in this paper is the participant's payment function. Equilibrium: refers to a combination of the best strategies of all participants, generally recorded as: $s^{*}=\left(s_{1}^{*}, \cdots, s_{i}^{*}, \cdots, s_{n}^{*}\right)$. The optimal strategy in equilibrium, it is the strategy that maximizes $u_{i}$ or $E_{u i}$ in all possible strategies of $i$. The comprehensive weight derived from formula (15) in this paper is the equilibrium of the game.

The single use of simple correlation function objectively empowers the evaluation indexes, and does not consider the impact of the evaluation indexes themselves on the importance of the evaluation results. In the actual evaluation process, the impact of the differences in the individual evaluation indexes on the final evaluation results is objective existence; single use of AHP for subjective empowerment is too subjective, only based on the subjective experience of decision makers to determine the weight, the influence of human factors is too large, ignoring the actual data and objective information. Therefore, combining the advantages of these two methods, the two kinds of empowerment are combined to obtain an integrated weighting method. However, the existing comprehensive weighting method is basically obtained by simply calculating the average of the subjective and objective weights or the weighted average, and the results are not reasonable enough. In this paper, the game theory method is used to combine the subjective and objective weighting methods to obtain the balanced comprehensive weights. The specific steps are as follows:

In order to improve the accuracy of multi-index object evaluation results, $L$ methods can be used to weight the indexes, thereby constructing a basic weight set $u_{k}=\left\{u_{k 1}, u_{k 2}, \cdots, u_{k m}\right\}, k=1,2, \cdots, L$. The arbitrary linear combination of the vectors $L$ is:

$$
u=\sum_{k=1}^{l} \alpha_{k} \cdot u_{k}^{\mathrm{T}},\left(\alpha_{k}>0\right)
$$

$u$ is a possible weight vector of possible weight sets, and $\left\{u \mid u=\sum_{k=1}^{l} \alpha_{k} \cdot u_{k}^{\mathrm{T}}\right\}\left(\alpha_{k}>0\right)$ represents a possible weight vectors set.

In order to select the most satisfactory weight $u^{*}$ from all possible weights, a balance should be found between all the different weights, even if the deviation between the ideal weight and each basic weight is minimized. Linear combination coefficients $\alpha_{k}$ in formula (6) are optimized, and the objectives of the optimization should be minimized dispersions in $\mathrm{u}$ and each of $u_{k}$, that is:

$$
\min \left\|\sum_{j=1}^{l} \alpha_{j} u_{j}^{\mathrm{T}}-u_{i}\right\|_{2},(i=1,2, \cdots, l)
$$


According to the matrix differential properties, the optimal first-order derivative condition of formula (12) can be transformed into the following linear equations:

$$
\left[\begin{array}{cccc}
u_{1} \cdot u_{1}^{\mathrm{T}} & u_{1} \cdot u_{2}^{\mathrm{T}} & \cdots & u_{1} \cdot u_{l}^{\mathrm{T}} \\
u_{2} \cdot u_{1}^{\mathrm{T}} & u_{2} \cdot u_{2}^{\mathrm{T}} & \cdots & u_{2} \cdot u_{l}^{\mathrm{T}} \\
\vdots & \vdots & \ddots & \vdots \\
u_{l} \cdot u_{1}^{\mathrm{T}} & u_{l} \cdot u_{2}^{\mathrm{T}} & \cdots & u_{l} \cdot u_{l}^{\mathrm{T}}
\end{array}\right]\left[\begin{array}{c}
\alpha_{1} \\
\alpha_{2} \\
\vdots \\
\alpha_{l}
\end{array}\right]=\left[\begin{array}{c}
u_{1} \cdot u_{1}^{\mathrm{T}} \\
u_{2} \cdot u_{2}^{\mathrm{T}} \\
\vdots \\
u_{l} \cdot u_{l}^{\mathrm{T}}
\end{array}\right]
$$

Calculation (13) is obtained and then normalized, that is:

$$
\alpha_{k}^{*}=\alpha_{k} / \sum_{k=1}^{l} \alpha_{k}, k=1,2, \cdots, l
$$

Then, the comprehensive weight is:

$$
u^{*}=\sum_{k=1}^{l} \alpha_{k}^{*} \cdot u_{k}^{\mathrm{T}}
$$

\section{Conclusion}

In the comprehensive evaluation of rural road maintenance quality, this paper uses the game theory method to combine the subjective weighting method (improved analytic hierarchy process) with the objective weighting method (simple correlation function method), solves the problem of subjective influence, and completely relies on the index data ignoring the characteristics of the evaluation index itself in the process of single weighting, making the determination of the weight more reasonable and the evaluation result more accurate. The evaluation results obtained by this evaluation method are clear, not only are the quality level of the road maintenance to be evaluated, but also the degree of deviation of the level given. Secondly, the rural highway quality evaluation is based on the extension theory of game theory. From multi-index and multi-angle, the number and category of influencing index factors are not limited, and the existing data and materials are used to a great extent. Therefore, the principle of the method is clear, the calculation is simple, and the computer is easy to perform programming calculation. This method is different from the method of calculating the rural road Maintenance Quality Index (MQI) in the specification, solves the problem of excessively relying on the weight recommendation value given by expert experience in the comprehensive evaluation, reducing the subjective influence in the individual evaluation index, and has opened up an effective method for comprehensive evaluation of rural road quality.

\section{Acknowledgements}

This paper is supported by the young innovative talents project of Guangdong ordinary universities (2017WQNCX066) and the scientific research project of Ruyuan local highway station: promotion service of Ruyuan X325 rural road pavement maintenance (2018-04-28). 


\section{Conflicts of Interest}

The authors declare no conflicts of interest regarding the publication of this paper.

\section{References}

[1] Chen, S., Saeed, T.U. and Labi, S. (2017) Impact of Road-Surface Condition on Rural Highway Safety: A Multivariate Random Parameters Negative Binomial Approach. Analytic Methods in Accident Research, 16, 75-89. https://doi.org/10.1016/j.amar.2017.09.001

[2] Wang, A.J. and Qiao, J.Z. (2013) Research on Rural Highway Maintenance Quality Evaluation System Based on Triangle Whitenization Weight Function. Advanced Materials Research, 639-640, 1275-1280.

https://doi.org/10.4028/www.scientific.net/AMR.639-640.1275

[3] Kumara, M.W., Gunaratne, M. and Lu, J.J. (2004) Methodology for Random Surface-Initiated Crack Growth Prediction in Asphalt Pavements. Journal of Materials in Civil Engineering, 16, 175-185.

https://doi.org/10.1061/(ASCE)0899-1561(2004)16:2(175)

[4] Transport Standardization (2009) Application on the Rural Road Maintenance Quality Evaluation Criterion in Jiangsu Province.

[5] Duan, Y.C., Liu, Y.-W. and Shan, F. (2011) Evaluation Model of High-Grade Highway's Maintenance Quality Based on AHP-FUZZY Algorithm. Highway, No. 2, 70-78.

[6] Zhang, G.L., Xiao, P., Zhang, R.-H., et al. (2015) The Research on AHP to Evaluate the Quality of Highway Maintenance Based on Extension Theory. Mathematics in Practice and Theory, 45, 31-38.

[7] Huang, F. (2014) Evaluation of Rural Highway and Bridge Maintenance Quality Based on Fuzzy Extension Theory. Journal of China \& Foreign Highway, 34, 357-361.

[8] Ma, L., Chen, H., Yan, H., et al. (2018) Post Evaluation of Distributed Energy Generation Combining the Attribute Hierarchical Model and Matter-Element Extension Theory. Journal of Cleaner Production, 184, 503-510. https://doi.org/10.1016/j.jclepro.2018.02.223

[9] Bo, L. (2017) Research on Safety Evaluation of Highway Alignment Based on Extension Theory. Highway, 62, 181-185.

[10] Bo, Y., Zhuo, C. and Li, H. (2017) Evaluation of Agri-Product Supply Chain Competitiveness Based on Extension Theory. Operational Research, No. 1, 1-28.

[11] Sutadian, A.D., Muttil, N., Yilmaz, A.G., et al. (2017) Using the Analytic Hierarchy Process to Identify Parameter Weights for Developing a Water Quality Index. Ecological Indicators, 75, 220-233. https://doi.org/10.1016/j.ecolind.2016.12.043

[12] Sun, L., Zhao, M., Shang, Y., et al. (2016) Research on Multi-Attribute Decision-Making in Condition Evaluation for Power Transformer Using Fuzzy AHP and Modified Weighted Averaging Combination. IET Generation Transmission \& Distribution, 10, 3855-3864. https://doi.org/10.1049/iet-gtd.2016.0381

[13] Asghari, M., Nassiri, P., Monazzam, M.R., et al. (2017) Weighting Criteria and Prioritizing of Heat Stress Indices in Surface Mining Using a Delphi Technique and Fuzzy AHP-TOPSIS Method. Journal of Environmental Health Science \& Engineering, 15, 1. https://doi.org/10.1186/s40201-016-0264-9 